\title{
Proposta de plano de cuidados de enfermagem para paciente queimado: estudo de caso
}

\section{Proposal for a nursing care plan for burned patient: case study}

\author{
Richardson Augusto Rosendo da Silva ${ }^{1} \bullet$ Vinicius Lino de Souza Neto ${ }^{2} \bullet$ Olga Alice Alencar Moreira ${ }^{3} \bullet$ \\ Fernanda Rafaela dos Santos ${ }^{4}$ - Rafael Medeiros dos Santos ${ }^{5}$ Thais Monteiro da Silva Padilha
}

\section{RESUMO}

A Sistematização da Assistência de Enfermagem é caracterizada por ser uma ferramenta utilizada pelo enfermeiro para aprimorar os saberes da prática, otimizando, aperfeiçoando e aprimorando as técnicas assistenciais a partir das novas evidências científicas. $O$ estudo teve como objetivo elaborar e validar um plano de cuidados para paciente queimado. Trata-se de um estudo de caso realizado no Centro de Tratamento de Queimados de um hospital público no nordeste do Brasil, no período de abril a maio de 2016. As etapas metodológicas do estudo foram estas: coleta de dados; elaboração dos diagnósticos, resultados e intervenções de enfermagem; elaboração e validação do plano de cuidados. Após a análise dos dados, foi realizada a elaboração e a validação de um plano de cuidados com quatro diagnósticos, resultados e 24 intervenções de enfermagem. Diante disso, o estudo permitiu identificar os diagnósticos, resultados e intervenções de enfermagem para a aplicação na prática clínica, corroborando para o cuidado científico e direcionado as necessidades prioritárias.

Palavras-chave: Processos de Enfermagem; Enfermagem; Queimaduras.

\section{ABSTRACT}

The Systematization of Nursing Care is characterized by being a tool that nurses use to improve the practical knowledge, optimizing, enhancing and improving the technical assistance will from the new scientific evidence. The study aimed to develop and validate a plan of care for burn patients. This is a case study in the Burn Treatment Center of a public hospital in northeastern Brazil, from April to May 2016. The methodological stages of the study were these: data collection; preparation of diagnoses / outcomes and nursing interventions; preparation and care plan validation. After analyzing the data produced and validated a care plan with four diagnostic / 24 results and nursing interventions. Thus, the study identified the diagnoses / outcomes and nursing interventions to implement in clinical practice.

Keywords: Nursing process; Nursing; Burns.

Professor Adjunto do curso bacharelado em enfermagem da Universidade Federal do Rio Grande do Norte - UFRN. E-mail: rirosendo@hotmail.com

2 Enfermeiro. Mestre em enfermagem pela Universidade Federal do Rio Grande do Norte. Especialista em Urgência e Emergência. Professor da graduação de enfermagem da UFRN/Departamento de Enfermagem/ Centro de ciências da Saúde. E-mail:vinolino@hotmail.com

${ }^{3}$ Discente do curso bacharelado em Enfermagem da Universidade Federal do Rio Grande do Norte- UFRN. E-mail: olguinhaalice@gmail.com

${ }^{4}$ Discente do curso bacharelado em Enfermagem da Universidade Federal do Rio Grande do Norte-UFRN.E-mail: fernanda_tinha@hotmail.com

${ }^{5}$ Discente do curso bacharelado em Enfermagem da Universidade Federal do Rio Grande do Norte- UFRN. E-mail: rafaelufrn@gmail.com

${ }^{6}$ Discente do curso bacharelado em Enfermagem da Universidade Federal do Rio Grande do Norte- UFRN. E-mail: thaismonst@hotmail.com

*Autor para correspondência: Vinicius Lino de Souza Neto; Universidade Federal do Rio Grande do Norte, Centro de Ciências da Saúde - Departamento de Enfermagem;

Campus Central, s/n, Lagoa Nova;59078-970 Natal - RN

${ }^{* *}$ Financiamento próprio 


\section{INTRODUÇÃO}

A Sistematização da Assistência de Enfermagem (SAE) é caracterizada por ser uma ferramenta utilizada pelo enfermeiro para aprimorar os saberes da prática, otimizando, aperfeiçoando e aprimorando as técnicas assistenciais a partir das novas evidências científicas. A implementação das práticas sistemáticas, seja no âmbito do setor público ou privado, requer do enfermeiro habilidades e competências para que transpasse as barreiras e sensibilize os demais profissionais ${ }^{1}$.

Assim, as ações e intervenções sistemáticas implicadas no processo de cuidar significam a mudança de uma assistência de enfermagem rotinizida para cientifica e instrumental no campo da pesquisa. Por isso, os cuidados sistemáticos destinados ao paciente queimado devem identificar os fenômenos de enfermagem para que, assim, sejam analisadas as necessidades prioritárias e realizadas as intervenções adequadas ${ }^{2}$. De acordo com o Ministério da Saúde (MS), no Brasil, ocorrem uma média anual de 1.000.000 acidentes por queimaduras em crianças $^{3}$.

O plano de cuidados é caracterizado como uma ferramenta da prática cotidiana do enfermeiro e permite o auxílio na organização do trabalho da equipe de enfermagem, visto que, além da otimização da prática, o plano de cuidados deve trabalhar em cima das necessidades humanas básicas, que são caracterizadas pelos aspectos ligados ao alimento, água, segurança, amor, todos elementos necessários para a sobrevivência e a saúde 4 .

O Conselho Internacional de Enfermagem (CIE), entendendo a necessidade e reconhecendo serem essenciais as inovações no âmbito da assistência, vem desenvolvendo a Classificação Internacional para a Prática de Enfermagem - CIPE ${ }^{\oplus}$, considerada um marco unificador dos diferentes sistemas de classificação dos elementos da prática profissional - diagnósticos, resultados e intervenções de enfermagem ${ }^{5}$.

$\mathrm{A} \mathrm{CIPE}^{\circledast}$ é um sistema de classificação estruturado no modelo dos sete eixos, sendo estes: foco, julgamento, meio, ação, tempo, cliente e localização. Para a elaboração dos diagnósticos, resultados, as afirmativas devem ser compostas por um termo do eixo foco e julgamento. Já as intervenções devem ter um termo do eixo ação e cliente, conforme regulamenta a ISO $18.104^{6}$.

Além disso, caracteriza-se por ser uma ferramenta que articula, na prática, o ensino e a pesquisa. Essa nova tecnologia da enfermagem corrobora para a complementação das práticas sistemáticas, permitindo ao enfermeiro uma olhar criterioso para as necessidades prioritárias na assistência à saúde 7 . Diante desse cenário, surgiu o seguinte questionamento: é possível elaborar e validar um plano de cuidados de enfermagem para pacientes queimados utilizando a CIPE $^{\circledR}$ ?. Assim, o estudo teve como objetivo elaborar e validar um plano de cuidados para paciente queimado.

\section{MÉTODO}

Trata-se de um estudo de caso realizado no Centro de Tratamento de Queimados (CTQ) de um hospital público no nordeste do Brasil, no período de abril a maio de 2016. $\mathrm{O}$ presente setor assiste crianças, adolescentes, adultos e idosos. A unidade da pediatria é composta por oito leitos, e conta com seis técnicos de enfermagem, quatro enfermeiros, dois médicos, e fisioterapeutas, que trabalham em regime de plantão. Assim, o estudo de caso corrobora com o ensino prático e científico da Enfermagem, predispondo a implementação e a avaliação de diversas ferramentas sistemáticas, dentre estas, o plano de cuidados ${ }^{8}$.

O estudo transcorreu nas seguintes etapas: coleta de dados por meio de instrumento adaptado e validado por enfermeiros especialistas e por profissionais da prática de enfermagem; a elaboração dos diagnósticos, resultados e intervenções de enfermagem da $\mathrm{CIPE}^{\circledR}$ versão 2015; elaboração e validação do plano de cuidados por enfermeiros especialistas.

A elaboração dos diagnósticos foi processual, ou seja, após a coleta de dados realizada por meio do instrumento validado, os pesquisadores identificaram os focos prioritários de enfermagem para que assim fossem elaborados os diagnósticos, resultados de enfermagem utilizando a CIPE $^{\oplus}$ versão 2015. Assim, estruturou-se uma proposta de plano de cuidados com os respectivos diagnósticos, resultados e intervenções de enfermagem que foi encaminhada por meio de planilhas utilizando o Excel for Windows para três enfermeiros da prática e dois docentes que pesquisam na área de sistematização hámais de cinco anos e por terem experiência e especialidade na clínica.

Os profissionais foram incumbidos de avaliar se as afirmativas propostas eram aplicáveis ao paciente. Assim, para análise do grau de concordância entre os especialistas, optou-se pelo índice de Kappa, analisado pelo programa StatisticalPackage for the Social Science (SPSS), versão 20.0. Foram consideradas validadas as afirmativas que obtiveram IC $\geq 0.80$.

Para isso, o estudo obteve certificação do Comitê de Ética e Pesquisa da Universidade Federal do Rio Grande do Norte, CAAE: 53307116.3.0000.5537 processo $n^{\circ}$ 1.450.346, respeitando assim a lei $n^{\circ} 466 / 12$.

\section{RESULTADOS E DISCUSSÃO}

Paciente, sexo feminino, 1 ano e 1 mês branca, 11 kg, residente do município de Assú do Rio grande do Norte. Mãe refere que a queimadura foi proveniente de um caldo de peixe, pegando a região anterior do tórax e MMII da criança. O primeiro atendimento (12/04/2016) foi realizado já na sala de pronto atendimento para queimados, sendo identificadas lesões de $2^{\circ}$ grau extensivas com aspecto bolhoso de tonalidade rósea e hiperemiada. 0 percentual corpóreo foi de $12 \%$ distribuídos em diversas regiões, 
sendo assim, sedada e realizados os curativos especiais. Já no dia 01/05/2016, a criança apresentou quadro de hipertermia $\left(39,3^{\circ} \mathrm{C}\right)$, leucocitose (14.000), adinamia, e pulsos periféricos e centrais irregulares, irritada e chorosa. Assim, a partir dos indicadores clínicos que a paciente apresentava, elaborou-se o plano de cuidados com os respectivos diagnósticos, resultados e intervenções da CIPE, conforme revela o Quadro 1.

Quadro 1. Proposta do plano de cuidados para paciente queimado

\begin{tabular}{|c|c|}
\hline $\begin{array}{c}\text { DIAGNÓSTICOS/ } \\
\text { RESULTADOS DE } \\
\text { ENFERMAGEM } \\
\text { IC }>0.80 \text { - Escores }\end{array}$ & $\begin{array}{l}\text { INTERVENÇÕES DE ENFERMAGEM } \\
\text { IC>0.80 - Escores }\end{array}$ \\
\hline $\begin{array}{l}\text { Integridade da pele } \\
\text { prejudicada }\end{array}$ & $\begin{array}{l}\text { Cuidar do sítio de inserção de } \\
\text { dispositivos invasivos; controlar } \\
\text { a ingestão e a excreta (balanço } \\
\text { hídrico); estimular a hidratação; } \\
\text { estimular a mudança de posição; } \\
\text { manter a pele limpa e integra; } \\
\text { estimular o aleitamento materno; } \\
\text { realizar suplementação nutricional } \\
\text { adequada; realizar o curativo e } \\
\text { avaliar a progressão. }\end{array}$ \\
\hline Risco para a infecção & $\begin{array}{l}\text { Avaliar o estado nutricional; avaliar } \\
\text { locais e inserção de cateteres } \\
\text { quanto presença de hiperemia; } \\
\text { manter vias aéreas permeáveis; } \\
\text { monitorar a temperatura e a } \\
\text { frequência respiratória; monitorar } \\
\text { os sinais e os sintomas de infecção } \\
\text { da queimadura; utilizar técnicas } \\
\text { assépticas apropriada na troca do } \\
\text { curativo e procedimentos invasivos. }\end{array}$ \\
\hline Hipertermia & $\begin{array}{l}\text { Evitar correntes de ar no ambiente; } \\
\text { evitar infusão de líquidos gelados; } \\
\text { manter o paciente hidratado; } \\
\text { monitorar cor e temperatura } \\
\text { corporal; monitorar nível de } \\
\text { consciência; monitorar ingestão e a } \\
\text { eliminação de líquidos; monitorar o } \\
\text { desequilíbrio de eletrólitos. }\end{array}$ \\
\hline Dor moderada & $\begin{array}{l}\text { Avaliar a dor quanto à localização, } \\
\text { frequência e duração; avaliar a } \\
\text { eficácia das medidas de controle da } \\
\text { dor por meio de um levantamento } \\
\text { constante da experiência de dor; } \\
\text { controlar os fatores ambientais } \\
\text { capazes de influenciar a resposta } \\
\text { do paciente ao desconforto (p.e.: } \\
\text { temperatura ambiente, iluminação, } \\
\text { ruído). }\end{array}$ \\
\hline
\end{tabular}

Fonte: adaptado de CIPE® (2015)

\section{DISCUSSÃO}

O diagnóstico de Enfermagem, Integridade da pele prejudicada foi caracterizado na paciente pela destruição tecidual presente na região anterior do tórax e nos MMII da criança. O sistema tegumentar é responsável pela termorregulação, e a homeostase da superfície da pele depende do equilíbrio da destruição celular e a renovação das células. Ressalva-se que a magnitude e duração das alterações fisiopatológicas das queimaduras são proporcionais à extensão da lesão podendo causar a instabilidade hemodinâmica e, consequentemente, alterações cardiovasculares, hidroeletrolíticos, pulmonares, renais, imunológicas e gastrointestinais ${ }^{9}$.

Para tentar atenuar as instabilidades e prevenir que o paciente entre em estado crítico da sua condição de saúde, o enfermeiro deve implementar intervenções imediatas em conjunto com a equipe de enfermagem. Por isso, o plano de cuidados propõe intervenções baseadas nas necessidades prioritárias dos pacientes, como, por exemplo, o estimulo à hidratação. As substâncias aquosas ajudam a manter a normovolemia, que deve ser controlada por meio do balanço hídrico ${ }^{9-10}$.

Além dessas intervenções, o enfermeiro pode fazer o uso da associação da sulfadiazina de prata, no sítio da lesão, e o medicamento fitoterápico à base de aloe vera, disponibilizado pelo SUS na forma farmacêutica de creme. Este fitoterápico é classificado como um anti-inflamatório não esteroide, no qual se destacam seu alto potencial cicatrizante, ação proliferativa, analgésica de feridas, além de otimizar a progressão tegumentar, promovendo melhores resultados no tempo e na qualidade do processo cicatricial ${ }^{10}$.

O risco para infecção é inerente à criança com queimadura, e decorre não só da destruição da pele, mas também das práticas assistências realizada pela equipe multiprofissional. A paciente apresentou um quadro de leucocitose, adinamia, hipertermia, caracterizando-se, assim, quadro de infecção sistêmica. A causa para este quadro está relacionada à multifatorialidade, dentre elas, o excesso de procedimentos invasivos aos quais os pacientes são submetidos durante a hospitalização como, por exemplo: punção venosa periférica, acesso venoso central, tubo orotraqueal, cateterismo vesical, traqueostomia e drenos em geral ${ }^{11}$

Para que isso não aconteça, o enfermeiro deve ser o agente transformador da realidade, com a criação de núcleos educacionais de âmbito multidisciplinar, a elaboração de protocolos operacionais e funcionais das técnicas, discussões clínicas sobre a segurança do pacientes, e também o monitoramento dos sinais vitais, que são indicadores quanto ao comportamento fisiológico; em crianças, qualquer tipo de evento, como a queimadura, a descompensação é nítida. ${ }^{11-12}$

A Hipertermia na paciente decorreu das lesões por queimaduras, que ocasiona uma resposta local e sistêmica, a depender da gravidade da área afetada. A resposta sistêmica ocorre devido à liberação de citocinas e outros mediadores na circulação. Em decorrência da ação de toxinas, o sistema talâmico é afetado aumentando a temperatura corpórea, podendo acarretar a desnaturação proteica e perda de grande quantidade de água ${ }^{12}$.

Desta forma, o enfermeiro, além de promover os cuidados prioritários evidenciados no plano, deve estar atento ao índice de proteína e promover a educação em saúde com relação à suplementação nutricional necessária para o processo de reabilitação e atenuação da febre. Ressalva-se que os alimentos ricos em ômega 3, 6 e 9, encontrados em 
peixes de águas frias, oleaginosas, óleos vegetais como de linhaça, gergelim e abacate, e azeite de oliva, desempenham um papel importante na redução do processo inflamatório. Além destes, alimentos probióticos, como os lactobacilos que têm propriedades anti-inflamatórias que estimulam o sistema imune, sendo encontrados em leites fermentados e outros produtos lácteos ${ }^{13}$.

Por fim, o diagnóstico de enfermagem de Dor, essa sensação nos pacientes queimados é evidenciada pela estimulação direta e lesão de nociceptores presentes na epiderme e na derme, transmitindo impulsos nervosos pelas fibras C e A-delta até o corno dorsal da medula espinal. É fundamental, durante o tratamento da dor, a conduta organizada que aborde a dor basal, a dor súbita e a dor decorrente do procedimento, com o objetivo de proporcionar ao paciente a sensação de conforto a partir de cuidados específicos e diferenciais ${ }^{14}$.

O manejo da dor consiste no tratamento farmacológico, com o uso de opioides, agentes anestésicos, antiinflamatórios não esteroides (AINE) e ansiolíticos. Além disso, o controle não farmacológico abarca o uso de técnicas de relaxamento, imagem orientada, balneoterapia, distração, hipnose, musicoterapia, toque terapêutico e técnicas de realidade virtual, que pode complementar o plano de cuidados ${ }^{15}$.

\section{CONCLUSÃO}

A partir dos dados apresentados, foi possível elaborar e validar um plano de cuidados com o uso da $\mathrm{CIPE}^{\oplus}$ versão 2015, composto por quatro diagnósticos, resultados e 24 intervenções de enfermagem. A identificação de tais diagnósticos pauta-se a partir das necessidades prioritárias da paciente, como também, os focos da prática de enfermagem. Nota-se que o plano de cuidados corrobora para um cuidado individual, cientifico, acolhedor e humano, proporcionado melhor qualidade de vida da paciente.

Além disso, as limitações do estudo voltam a ser desenvolvidas em uma determinada área geográfica e também uma clientela especifica. Porém, impera-se que novos estudos devem ser desenvolvidos em outras realidades, para que possa dimensionar o cuidado de enfermagem aos pacientes queimados.

\section{REFERÊNCIAS}

1. Santos WN. Sistematização da assistência de enfermagem: o contexto histórico, o processo e obstáculos da implantação. J Manag Prim Health Care [Internet]. 2014; 5(2):153-58. Disponível em: www.jmphc.com.br/saude-publica/index.php/ jmphc/article/download/210/213.

2. Mukerji G, Chamania S, Patidar GP, Gupta S. Epidemiology of pediatric burns in Indore, India. Burns [Internet]. 2001; 27(1):33-8. Disponível em: https://www.ncbi.nlm.nih.gov/ pubmed/11164662.

3. Montes SF, Barbosa MH, Neto ALS. Aspectos clínicos e epidemiológicos de pacientes queimados internados em um Hospital de Ensino. Rev Esc Enferm USP [Internet]. 2011;
45(2):369-73. Disponível em: http://www.revistas.usp.br/ reeusp/article/viewFile/40710/43983.

4. Regis LFLV, Porto IS. Necessidades humanas básicas dos profissionais de enfermagem: situações de (in)satisfação no trabalho. Rev Esc Enferm USP [Internet]. 2011; 45(2):33441. Disponível em: http://www.scielo.br/pdf/reeusp/v45n2/ v45n2a04.pdf.

5. Garcia TR, Nóbrega MML. A terminologia CIPE e a participação do centro CIPE brasileiro em seu desenvolvimento e disseminação. Rev Bras Enferm [Internet]. 2013; 66(esp):14250. Disponível em: https://grupos.moodle.ufsc.br/pluginfile. php/178459/mod_resource/content/1/A\%20terminologia\%20 CIPE\%20e\%20a\%20participa\%C3\%A7\%C3\%A3o\%20do\%20 Centro\%20brasileiro.pdf.

6. Garcia TR. (Org.). Classificação Internacional para a Prática de Enfermagem $\mathrm{CIPE}^{\oplus}$ : aplicação à realidade brasileira. Porto Alegre: Artmed; 2015.

7. Nóbrega RV, Nóbrega MML, Silva KL. Diagnósticos, resultados e intervenções de enfermagem para crianças na Clínica Pediátrica de um hospital escola. Rev Bras Enferm [Internet]. 2011; 64(3):501-10. Disponível em: http://www.scielo.br/pdf/ reben/v64n3/v64n3a14.pdf.

8. Galdeano LE, Rossi LA, Zago MMF. Roteiro instrucional para elaboração de um estudo de caso clínico. Rev Latino-am Enfermagem [Internet]. 2003; 11(3): 371-5. Disponível em: http://www.scielo.br/pdf/rlae/v11n3/16548.

9. Suzanne C. Smeltzer et al.; Brunner \& Suddarth, tratado de enfermagem médico-cirúrgico- Rio de Janeiro: Guanabara Koogan, 2012.

10. Ferreira FV, Paula LB. Sulfadiazina de prata versus medicamentos fitoterápicos: estudo comparativo dos efeitos no tratamento de queimaduras. Rev Bras Queimaduras [Internet]. 2013; 12(3):132-9. Disponível em: Lildbi.bvs.br/ lildbi/docsonline/get.php?id=141.

11. Salomé GM. Diagnóstico de enfermagem dos pacientes internados em uma Unidade de Terapia Intensiva. Saúde Coletiva [Internet]. 2011; 8(47):24-8. Disponível em: http://www.redalyc.org/articulo.oa?id=84217101006.

12. King MA, Clanton TL, Laitano O. Hyperthermia, dehydration, and osmotic stress: unconventional sources of exercise-induced reactive oxygen species. Am J Physiol Regu llntegr Comp Physiol [Internet]. 2016; 310:105-114. Disponível em: https://www.ncbi.nlm.nih.gov/pubmed/26561649.

13. Vidal AM, Dias DO, Martins ESM, Oliveira RS, Nascimento RMS, Correia MGS. A ingestão de alimentos funcionais e sua contribuição para a diminuição da incidência de doenças. Cadernos de Graduação - Ciências Biológicas e da Saúde [Internet]. 2012; 1(15):43-52. Disponível em: https://periodicos.set.edu. br/index.php/cadernobiologicas/article/viewFile/284/112.

14. Calil AM, Pimenta CA. Importância da avaliação e padronização analgésica em serviços de emergência. Acta Paul Enferm [Internet]. 2010; 65(1):132-8. Disponível em: http://www. producao.usp.br/handle/BDPI/4018.

15. Castro RJA, Leal PC, Sakata RK. Tratamento da dor em queimados. Rev Bras Anestesiol [Internet]. 2013; 63:15458. Disponível em: http://bases.bireme.br/cgi-bin/wxislind. exe/iah/online/?IsisScript=iah/iah. $x$ is \& src=google \&base= LILACS $(=p$ \&nextAction $=$ lnk \& exprSearch $=666127$ \& indexSearch $=$ ID. 